\title{
REaCH-Resiliency Engagement and Care in Health; A Telephone Befriending Intervention to Address The Psycho-Social Challenges of Vulnerable Population in The Context of COVID-19 Pandemic: An Exploratory Trial in India
}

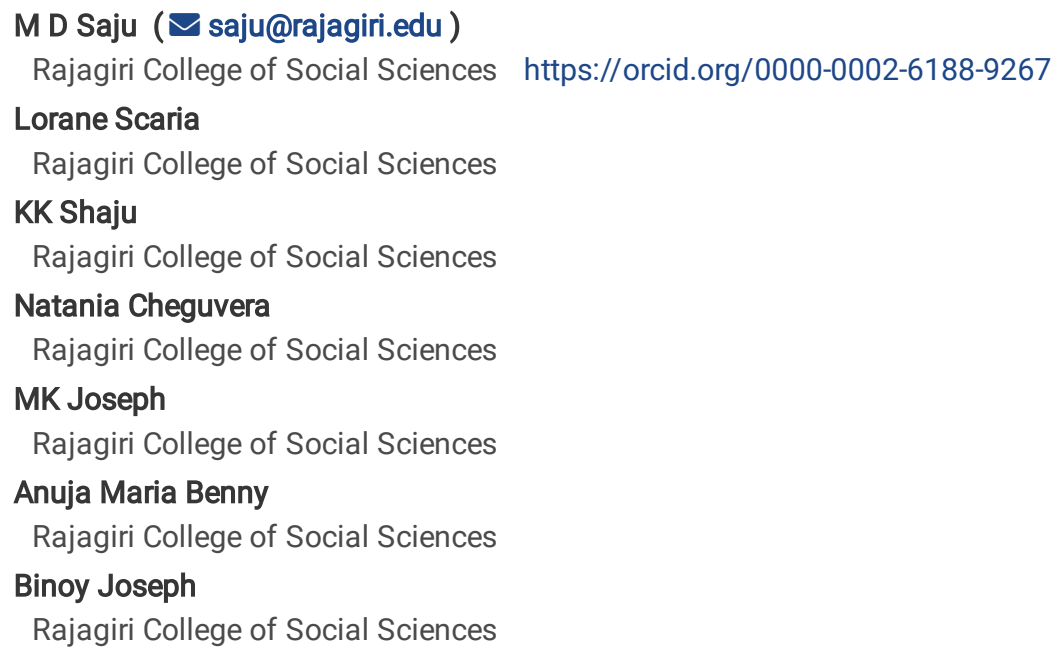

License: @ (7) This work is licensed under a Creative Commons Attribution 4.0 International License. Read Full License 


\section{Abstract}

Background Observational evidence suggests that befriending interventions provided by the lay mental health workers, when trained and supervised by experts, are effective in enhancing social support, wellbeing and reducing depression. We carried out a telephone intervention for youth, recruited from DDUGKY (Deen Dayal Upadhyaya Grameen Kaushalya Yojana), a central government funded skill development project in India.

Methods We performed an exploratory trial in Kerala, India, between $29^{\text {th }}$ July, 2020 and $26^{\text {th }}$ August, 2020 with pass out students of DDUGKY. From a total of 1036 students, 498 fulfilled the criteria and were recruited for the program. We randomized the participants via a computer generated randomization list. Out of 498 eligible participants, 251 were randomized to the intervention group and 247 to the general enquiry call group. Total of 439 (89\%) participants, (251 (100\%) = intervention arm and $188(76 \%)$ = control arm), completed follow-up assessment after one month. The primary outcomes of the study included depression, measured by Patient Health Questionnaire (PHQ-9) and wellbeing, using WHO Wellbeing Index; while secondary outcomes included social support from friends and family.

Results Analysis was done for 439 pass out students who completed one-month intervention. Befriending intervention was effective in increasing the wellbeing $(p=0.000)$ and social support $(p=0.008)$ in the intervention group. Regression analysis revealed that befriending intervention is associated with notable changes in support from significant others (OR: 2.91, $P=0.005)$, support from family (OR: 2.47, $P=0.013$ ) and support from friends (OR: 2.52, $\mathrm{P}=0.007)$.

Conclusion Befriending intervention was found successful in improving overall wellbeing and social support among student participants of the programme. Large-scale transformational efforts to secure country's mental health service pipeline is possible by integrating this intervention into existing DDU-GKY institutional and program eco-systems. Task shifting and sharing strategies can be effectively employed to improve the mental health outcomes of the people especially during a community crisis.

Trial Registration Clinical Trial Registry India; ICMR-NIMS (Registration Number: CTRI/2020/07/026834), prospectively registered (Registration date: $27^{\text {th }}$ July 2020) , http://ctri.nic.in/Clinicaltrials/showallp.php?mid1=45953\&EncHid=\&userName=CTRI/2020/07/026834

\section{Background}

The COVID-19 pandemic exerts a long term impact on mental health globally, given the medical, economic and social implications the crisis has generated. Pandemic related lockdown has become one of the most stressful happenings due to unpredictability and uncertainty of the situation [1]. Health emergencies demand for physical health interventions but fail to look at other aspects in social, emotional and economic domains, which is a prerequisite for wellbeing. Ignoring these domains may result in irreparable damages. Evidence shows that socio economic vulnerability is an important predictor of common mental disorders [2-5] mainly due to inadequate social support in the form of emotional, informational and practical resources [6] and limited access to social and health services [7]. It has also been proven that strengthening social support by investing on available social networks, delivered through significant people in one's life, might be a feasible method to promote the social, emotional and economic outcomes among people [8].

We searched a few significant data bases (Embase, Medline, Psycinfo, PubMed and Google Scholar), using pertinent key words including; lay health workers driven telephonic befriending interventions for wellbeing and coping in specific disasters / epidemics/ pandemics/ and economic recessions. Searches were updated on a regular basis to ensure latest developments in the context of Covid-19 were not missed out. We were unable to identify any lay health workers driven telephone befriending studies focusing on vulnerable populations that have sought to enhance wellbeing especially in the time of health crisis.

There is a paucity of research that evaluates the mental health outcomes during this pandemic. As far as we know, this is the first study to introduce a low cost intervention model customized to address the specific needs of a vulnerable group. Intervention models which utilized the existing human resources, using task shifting and task sharing strategies, were found to be effective especially in times of crisis [9]. This strategy is particularly beneficial when local resources are generally overburdened and outside support is urgently required [7]. It is for the first time that the combination of four domains of intervention; proactive engagement, crisis intervention, brief problem solving oriented supportive therapy and assertive engagement with community resources were used in a befriending model. We postulated that Resiliency Engagement and Care in Health $(\mathrm{REaCH})$ intervention would be more effective than general enquiry telephone calls for this specific population for improvements in social support, symptoms of depression and wellbeing outcomes. In this exploratory trial paper, we outline the effectiveness of a pilot exploratory trial conducted on 439 student participants of Rajagiri DDU-GKY centre, during the time of COVID - 19, pandemic community crisis.

\section{Methods}

\section{Overview}


REaCH; a "Befriending" intervention, was provided to the pass out students of Deen Dayal Upadhyaya Grameen Kaushalya Yojana (DDU-GKY) centre of Rajagiri College of Social Sciences (RCSS), in Kerala, India. DDU-GKY is an initiative of the Ministry of rural development (MoRD), Government of India (GOI), which was launched in 2014 (http://ddugky.gov.in). The objective of this initiative is to add diversity to the incomes of the rural poor families and to cater to the career aspirations of rural youth. The focus of this project is rural youth aged 15 to 35 years from poor families. DDU-GKY has its branches in 28 States of India. Currently, there are 1,575 projects being implemented by over 771 partnering institutions. The aim of the intervention is to provide additional social support through the development of a non-judgmental, affirming, emotionfocused relationship over time that is provided free of cost by the DDU-GKY staff.

The $\mathrm{REaCH}$ intervention was designed to reduce depression and promote wellbeing through mobilization of social support from significant others, family and friends. It aims to deliver a three phase intervention to pass out students of DDU-GKY centre of RCSS and to evaluate its effectiveness in modulating the depression and wellbeing. It also aims to estimate the importance of social support in mediating the positive and negative outcomes of health crises. Detailed study protocol is submitted for publication separately (Reference Number: TRLS-D-20-01106).

\section{Study Design}

We conducted an exploratory trial of REaCH intervention, between 29th July, 2020 and 26th August, 2020. The trial randomized participants to a structured befriending intervention or a general enquiry phone call based on computer generated random numbers. There will be two data collection points; before treatment and one-month post baseline. REaCH intervention was motivated by the success of an empathetic engagement of the DDU-GKY staff of RCSS with their alumni in the context of lockdown due to Covid-19 pandemic. The purpose was to understand the varied concerns of students during COVID-19 lockdown. The feedback from the participants suggested that most of them were in need of some kind of support to cope up with the situation. Quantitative and Qualitative data collected from the students further informed the intervention components. We undertook further pilot work to align their needs and intervention components, to develop processes and to find the operational challenges.

\section{Study Participants}

The participants of this two armed pilot intervention study were recruited from Rajagiri DDUGKY centre. Broad inclusion criteria were used in the participant recruitment, i.e., the pass out students, who were already placed in some jobs or in search of jobs; currently working or not working were included in the study. The recruitment of participants happened with the baseline assessment using google forms. The follow-up assessment was done using the same instruments to evaluate the effectiveness of the telephonic befriending intervention program. Out of 1036 potential participants from the centre, 538 participants were excluded due to reasons such as not meeting the inclusion criteria or were not interested to participate in the study. Out of 498 students enrolled, 251 were randomized to the intervention group for the structured befriending intervention and general enquiry telephone call was provided for the rest. 439 (43\%) respondents were included for the final analysis. Recruitment and intervention was conducted over a span of one month, where the end date of the pilot study was one month after the first session of the intervention (See Fig 1)

\section{Intervention}

\section{Training of the staff}

An important feature of this study was task-sharing and task-shifting through rigorous training and systematic supervision. Intervention team consisted of staff who were part of DDUGKY Project for a minimum of one year. Prelude to the intervention, the REaCH intervention team staff received one day ( 6 hours) training. The training consisted of the content and process of intervention. In addition to the training, an intervention manual, video of the training material, audio clips of sample interviews and a module on frequently asked questions were provided to the REaCH intervention team. The intervention manual has guidelines on developing relationships with clients, introduction and orientation to befriending, management of participant distress, confidentiality and safety issues for both staff and participants. Training and discussions between the project team and the staff members were facilitated through online platforms.

Two-layer supervision was introduced to ensure fidelity to the protocol: In the first level, the staff was supervised by two non-medical mental health professionals (Psychiatric social worker and a Clinical psychologist) working in the agency. In the second level, the mental health supervision was performed through a Psychiatrist, two Psychiatric social workers and two Clinical psychologists from RCSS. Regular supervisory meetings were conducted once in a week to collect the feedback from the intervention providers.

\section{Randomization}

Participants were randomly assigned in 1:1 ratio, via computer generated random number list for the structured telephone befriending intervention or general enquiry telephone calls. The odd numbers were allotted to the intervention arm and even numbers to the control arm. The principal investigator, trial team, trial manager and the staff members were blinded to the allocation codes during the trial. The recruitment was 
done by a computer technician. Randomization list was password protected and had not been shared with anyone involved in the study. Use of computer based data allocation helped in masking the outcomes from the intervention providers. The group allocation of the participants was masked by introducing general enquiry telephone calls with the control group. To eliminate contamination, we separated the structured intervention team and general enquiry team physically and concealed the group to which they belonged and the type of instruction they received. Lock down related social distancing and work from home mode of functioning of the staff made this masking easy. A committee of professionals and a team of researchers provided additional oversight to the trial.

\section{Intervention arm: Structured Befriending Intervention}

Semi-structured Intervention manual was used to allow sufficient flexibility to suit the needs of each participant. REaCH intervention consisted of three phases. In Phase 1, participants were assessed for various psycho-somatic and social indicators of distress such as sleep, appetite, interpersonal relationships, adjustments and work life to determine the level of disturbance. First level intervention consisted of proactive engagement and crisis intervention which focused on psycho-education, self-absorbing activity engagements and symptom based intervention. In phase 2, Brief problem-solving support oriented therapy was provided with specific focus on their current felt needs and problems. Prioritized needs were targeted through mobilization of untapped resources. Phase 3 focused on: assertive linkage with available community resources and introduce preventive strategies; linking them with employment opportunities and community resources; also to sensitize about mental health needs and psychological capacity building to deal with future challenges. These three phases of intervention were spread across four sessions of $30 \mathrm{~min}$ to 1 -hour duration.

\section{Control arm: General enquiry phone calls}

Participants randomised to the control arm were not given any intervention. They received 4 general enquiry phone calls lasting 5 to 30 minutes. General inquiry dealt with precautions that need to be taken to protect themselves from the pandemic, and the ways of coping with the lockdown related issues. Main focus of the phone call was on psycho-education based inquiries on COVID-19. No specific training was given for the staff making the telephonic calls in the control arm.

\section{Study Tools}

\section{Assessments and Procedures}

For assessments, an online questionnaire of quantitative tools was prepared using Google forms, with a consent form appended to it. Participants accessed the survey links through online communication platforms like Email or WhatsApp. After accepting the informed consent sheet, the participants were auto directed to the demographic information questionnaire and other standardised tools in a sequential manner. Questionnaire included Patient Health Questionnaire (PHQ-9) [10], Multidimensional scale of perceived social support (MSPSS) [11], WHOwellbeing index 5 [12] and sociodemographics. These questionnaires were translated into the local languages and reverse-translated for accuracy. Detailed instructions and sufficient explanations were provided in the initial page of the online survey.

\section{Outcome measures}

The demographic variables were age, gender, marital status, occupation, education level and colour of ration card. Presence of Depression was measured using the PHQ-9, with scores of 1-4, 5-9, 10-14, 15-19, 20-27 indicating minimal, mild, moderate, severe and extreme depressive symptoms. Wellbeing was measured using WHO-5. The total row score ranging from 0 to 25 is multiplied by 4 to provide the final score. 0 represents the worst possible wellbeing and 100 represents best possible wellbeing. The MSPSS measured perceived social support from three sources: family, friends, and a significant other. This measure contains 12 questions which were rated in a 7-point scale as "Very Strongly disagree", "Strongly disagree”, “Mildly disagree”, “Neutral”, "Mildly agree”, "Strongly agree”, "Very strongly agree”. The MSPSS yielded high internal consistency ( $a=0.88$ ), stability (yielded $a=0.85$ after 3 months from first administration) and moderate construct validity as the SS scores were negatively correlated to anxiety $(r=-0.18 ; p<0.01)$ and depression scores [13]. All the measures used were cross culturally validated for sensitivity and reliability [14-17]. Post-intervention follow-up assessment was carried out through an online platform link for both control and intervention arm. This used the same baseline survey assessment tools and was performed online.

\section{Statistical Analysis}

A target sample size of 490 participants (245 in each group) was estimated to provide $85 \%$ power and to generate a two-sided p value of 0.05 (alpha=5\%). Our study included 251 participants in the intervention and 248 participants in the control after randomization. At follow up, 251 from intervention and 188 from control, completed post assessment. All participants were included in the analysis according to their allocated group at randomisation. Statistical tests used a p value less than 0.05 for significance. All statistical analysis procedures were done using STATA 14 and R version 3.6.3. Baseline summary statistics (mean, standard deviation, percentage) were calculated based on groups. Chi-square tests, T-tests were performed to test the significance between the study variables. Odds ratio of the outcome variables for the post-assessment 
were calculated using logistic regression modelling and $95 \%$ confidence intervals were presented. We analysed descriptive summaries of sociodemographic aspects and the scores of Wellbeing -5, PHQ- 9 and MSPSS as the baseline and after a month.

No additional human resource expense was incurred for the study, as we have utilized the services of the existing staff of the project. The intervention development services and training of the staff was done voluntarily. The only additional cost was, the telephone and internet charges which was negligible as most of the staff have been subscribed to the unlimited outgoing call plan. The intervention manual and frequently asked questions were e-content, so printing and stationery costs were also minimal. Our account statement showed that only less than INR 20 (it is around one fourth of a US dollar) was spent on each student for the intervention.

\section{Results}

The study included 439 participants. At baseline, the mean age of the participants was 25 years (S. D = 5.7). Majority of the participants were females (64.2\%), unmarried (63.55\%) and hailed from economically poorer households (57.63\%). $42.8 \%$ of the respondents were either unemployed or housewives even though most of the participants had completed minimum education to be employed (91.2\%) (Table 1). Intervention and usual treatment groups were similar in terms of gender, occupation, education and marital status. At baseline, $8.2 \%$ people were recorded with moderate and above depression and $55 \%$ of them were females and $80.6 \%$ of them were below the age of 30 . Mean score of wellbeing index by $\mathrm{WHO}$ in the current study was $15.6 \pm 5.5$, while the mean score of the perceived social support scale was $65 \pm 13.4$. Mean scores of the subscales of social support were significant others $(21.8 \pm 4.8)$, family $(22.2 \pm 4.8)$ and friends (20.9 \pm 5.01$)$ respectively. 
Table 1

Participant Characteristics of Intervention and Control groups at Baseline

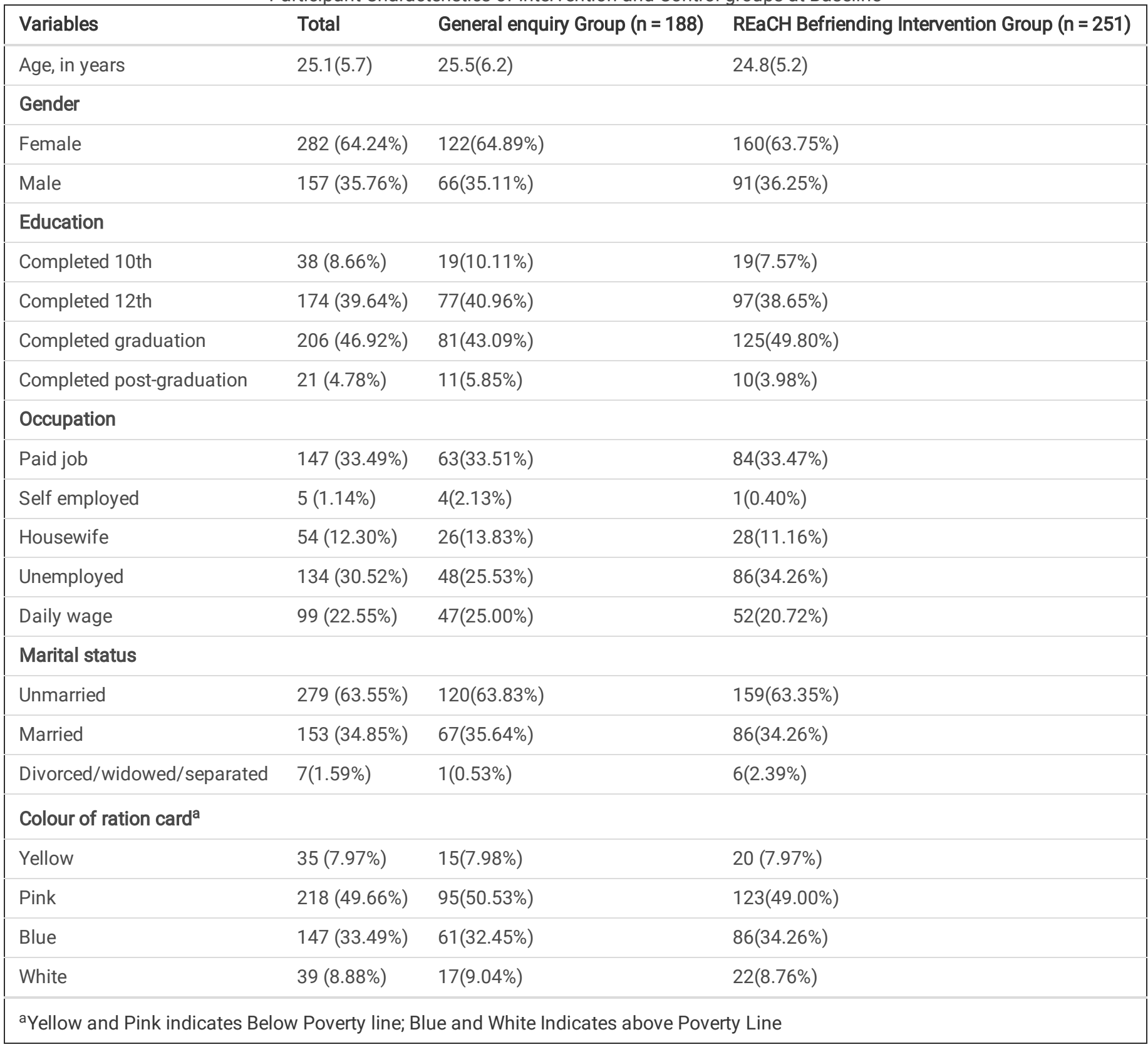

Mean scores of wellbeing, depression and social support for the intervention group at baseline were 15.6, 4.83 and 65.17 which changed to 17.4, 4.64 and 67.7 respectively after the befriending intervention. Paired sample t test was employed to measure the significant difference between intervention and treatment groups after the intervention. Befriending intervention significantly increased wellbeing ( $p=0.000)$ and social support (0.008) in the intervention group (Table 2). However, decreased depression scores for the intervention group were not statistically significant. 
Table 2

Outcome Measures; Wellbeing, Depression and Social Support between Befriending Intervention group and general enquiry group

\begin{tabular}{|c|c|c|c|c|c|c|c|c|}
\hline & \multicolumn{3}{|c|}{ REaCH - Befriending intervention } & \multicolumn{3}{|c|}{ General enquiry intervention } & \multicolumn{2}{|c|}{$\begin{array}{l}\text { REaCH model v/s General enquiry } \\
\text { model }\end{array}$} \\
\hline & Mean (S.D) & $\mathrm{T}(\mathrm{df})$ & $P$ & Mean (S.D) & $\mathrm{T}(\mathrm{df})$ & $P$ & OR (95\% Cl) & $P$ \\
\hline \multicolumn{9}{|l|}{ Wellbeing } \\
\hline Baseline & $15.64(5.75)$ & \multirow{2}{*}{$\begin{array}{l}-4.28 \\
(231)\end{array}$} & \multirow[t]{2}{*}{0.000} & $15.33(5.15)$ & \multirow{2}{*}{$\begin{array}{l}-2.35 \\
(145)\end{array}$} & \multirow[t]{2}{*}{0.010} & & \\
\hline $\begin{array}{l}\text { Post - } \\
\text { assessment }\end{array}$ & $17.41(4.90)$ & & & $16.71(5.38)$ & & & $1.29(0.85,1.96)$ & 0.224 \\
\hline \multicolumn{9}{|l|}{ Depression } \\
\hline Baseline & $4.83(3.56)$ & \multirow{2}{*}{$\begin{array}{l}0.60 \\
(231)\end{array}$} & \multirow[t]{2}{*}{0.727} & $4.88(3.47)$ & \multirow{2}{*}{$\begin{array}{r}-0.73 \\
(145)\end{array}$} & \multirow[t]{2}{*}{0.231} & & \\
\hline $\begin{array}{l}\text { Post - } \\
\text { assessment }\end{array}$ & $4.65(3.57)$ & & & $5.12(4.21)$ & & & $0.60(0.30,1.17)$ & 0.132 \\
\hline \multicolumn{9}{|c|}{ Social Support } \\
\hline Baseline & $\begin{array}{l}65.18 \\
(14.10)\end{array}$ & \multirow{2}{*}{$\begin{array}{l}-2.4 \\
(231)\end{array}$} & \multirow[t]{2}{*}{0.008} & $\begin{array}{l}64.92 \\
(13.01)\end{array}$ & \multirow{2}{*}{$\begin{array}{l}3.47 \\
(145)\end{array}$} & \multirow[t]{2}{*}{0.999} & & \\
\hline $\begin{array}{l}\text { Post - } \\
\text { assessment }\end{array}$ & $\begin{array}{l}67.70 \\
(13.50)\end{array}$ & & & $\begin{array}{l}61.68 \\
(13.84)\end{array}$ & & & $2.62(1.71,4.02)$ & 0.000 \\
\hline
\end{tabular}

The difference in mean was significant for the social support scores and the social support improved (mean difference $=2.52$ ) for the REaCH Befriending Intervention group compared to the General enquiry intervention group. Wellbeing (mean difference $=1.78)$ and depression (mean diff $=-19)$ recorded some changes between baseline and post intervention scores, however the differences were not statistically significant. Participants in the REaCH intervention group had 2.62 times higher social support at follow-up (OR: 2.62, $\mathrm{P}=0.000)$.

Table 3 represents Odds ratio, 95\% Confidence Intervals and $P$ Values from logistic regression models along with difference in mean values of the sub scales of social support. Regression analysis revealed that befriending intervention was associated with notable changes in support from significant others (OR: 2.91, $\mathrm{P}=0.005)$, support from family (OR: $2.47, \mathrm{P}=0.013$ ) and support from friends (OR: $2.52, \mathrm{P}=0.007)$. Suicidality and Depression was lower and wellbeing was higher for the intervention group, however the results were not statistically significant.

Table 3

Social Support from Significant others, Family and Friends-Odds Ratio, 95\% Cl of Odds ratio, and p values.

\begin{tabular}{|c|c|c|c|c|}
\hline & \multirow{2}{*}{$\begin{array}{l}\text { REaCH - Befriending } \\
\text { intervention } \\
\text { Mean (S.D) }\end{array}$} & \multirow{2}{*}{$\begin{array}{l}\text { General enquiry } \\
\text { intervention }\end{array}$} & \multicolumn{2}{|c|}{$\begin{array}{l}\mathrm{REaCH} \text { model v/s General enquiry } \\
\text { model }\end{array}$} \\
\hline & & & Odds Ratio $(95 \% \mathrm{Cl})$ & $\mathrm{P}$ \\
\hline \multicolumn{5}{|c|}{$\begin{array}{l}\text { Social support - significant } \\
\text { others }\end{array}$} \\
\hline Baseline & $21.76(5.14)$ & $21.94(4.54)$ & & \\
\hline Post - assessment & $21.49(5.31)$ & $19.98(6.94)$ & $2.91(1.38,6.15)$ & 0.005 \\
\hline \multicolumn{5}{|c|}{ Social Support-Family } \\
\hline Baseline & $22.22(5.18)$ & $22.24(4.44)$ & & \\
\hline Post - assessment & $21.62(5.45)$ & $20.01(6.85)$ & $2.47(1.21,5.06)$ & 0.013 \\
\hline \multicolumn{5}{|c|}{ Social support- Friends } \\
\hline Baseline & $21.20(5.05)$ & $20.75(5.21)$ & & \\
\hline Post - assessment & $20.76(5.41)$ & $18.98(6.98)$ & $2.52(1.28,4.96)$ & 0.007 \\
\hline
\end{tabular}

\section{Discussion}

This paper explored the feasibility, acceptability and effectiveness of a structured befriending intervention by the staff members over a general enquiry call intervention for reduced depression, enhanced wellbeing and social support. The structured Befriending intervention modestly improved wellbeing and significantly improved the social support and mildly reduced the depression scores among the participants. There was also a focus on exploring both the staff' and participants' perceptions on effectiveness and acceptability of this intervention program. 
Our findings are in line with the previous studies which showed reduced depression, enhanced wellbeing and social support using befriending interventions. Befriending intervention by non-specialist workers have been proven to be effective in tackling mental health concerns [18-20]. Also, similar to our pilot study findings, a meta-analysis by Mead et.al. (2010) [21] on the impact of befriending on depressive symptoms, emotional distress and social support, found a modest effect in varied patient groups including individuals with prostate cancer or dementia. Another meta-analysis by Siette et.al. (2017) [22] revealed that befriending was significantly associated with better patient-reported outcomes measures such as quality of life, increased social support and decreased loneliness.

For the first time, the befriending intervention was modelled with four specific components; such as, Proactive engagement, crisis intervention, Brief Problem-solving Support Oriented Therapy and assertive linkages. To the best of our knowledge, limited studies employed structured telephone befriending intervention to manage psychological and social determinants of vulnerable groups, particularly for the youth from lowincome families. After our pilot study, we realized the need for making task-sharing and task-shifting strategies more acceptable and effective. We proposed to strengthen task-sharing by providing certain supports: a) increased number of mental health professionals to supervise and mentor the intervention providers; b) two layer structured supportive supervision; and c) adequate training and standardization of the intervention. Adequate training and effective supervisory mechanism of the befrienders is the key to ensure successful delivery of interventions [25] (Mendenhall et.al. 2014). Considering the restrictions posed by the pandemic and the transformational leap in technology, the staff were systematically trained for 6 hours of virtual classroom tutoring. Ensuring the fidelity to the protocol came out as a major barrier; so strategies like sharing the online training videos, printed protocol manual, printed frequently asked questions and setting up a centre to provide continuous online supervisory support were brought about to help DDUGKY functionaries to implement the intervention effectively. This has also minimized the variability between providers. To meet the pre-specified quality assurance and fidelity standards, they were supervised by intervention coordinators and mental health team.

\section{Strengths and Limitations of the Study}

Task-shifting and task-sharing between lay personnel are already proven to improve access to health care services and specifically improve mental health [23]. In addition, the use of lay health workers in the care of subjects with common mental disorders was not only found costeffective but also cost-saving. [24]. Thus by the concept of delivery of intervention by DDU-GKY staffs, we enhance the use of existing staff for now and future; reduce cost; improve sustainability and increase acceptability. Interventions through these staff are particularly beneficial as these staff have the advantage of being better informed regarding the issues of the students.

Volunteerism and task shifting strategies made this intervention most cost effective. The only cost incurred for the intervention was the telephone and internet charges, which was negligible. The intervention manual and frequently asked questions were e-content, so printing and stationery costs were also minimal. Impact of REaCH intervention has to be looked at from the changes in the control group scores, where the control group had significant increase in negative outcomes and reduction in positive outcomes such as social support and wellbeing. Our study had its limitations as well. Generalizability of the study is limited as the sample is rural youth recruited from a DDUGKY centre in Kerala. Short follow up period was also considered as a limitation of the current trial.

\section{Conclusion}

In the global mental health context, the findings strengthen the case for adoption of the structured befriending intervention through nationwide network of DDUGKY centres as a mental health promotion strategy. Replicating the intervention content and process across India would benefit more than 1.2 million semi-skilled professionals passed out from different Program Implementation Agencies, under this scheme. Though the intervention was individual focused, in the collectivist context of India, intervention impact extends to their families and even to their extended families. At individual level, $\mathrm{REaCH}$ extends emotional support, educates on strategies to alleviate crisis and offer practical support and opportunities (e.g., resuming jobs) during the time of pandemic. At population level, we also address the psychological and social determinants that are likely to improve mental health and reduce inequities in families and thereby society. At policy level, we aim to significantly affect new programmatic policy and clinical guidelines that will lead to improvements in the psychological and social needs of economically backward and vulnerable groups. Ultimately, we aim to make social and health services accessible and affordable to all, consequently, reduce the mental health gap, improve social support systems and overall wellbeing of the students.

\section{Abbreviations}

COVID-19: coronavirus disease 2019

WHO-5: World Health Organization-Five Well-being Index

PHQ-9: Patient Health Questionnaire

MSPSS-12: Multidimensional Scale of Perceived Social Support 
DDUGKY: Deen Dayal Upadhyaya Grameen Kaushalya Yojana

NIRDPR: National Institute for Rural Development and Panchayath Raj

PIA: Project Implementing Agency Declarations

\section{Declarations}

\section{Ethics Approval and Consent to Participate}

The purpose, objectives and other information were provided in the landing page of the mobile link. We enrolled the participants in the intervention phase after receiving their oral consent, explaining the intervention objectives and brief description of the purpose, content, implications and risks of participation. Verbal consent was obtained from the participants as obtaining written consent was not feasible due to current pandemic situation. Ethics committee approval for the exploratory trial was obtained from the Rajagiri Institutional Review Board (IRB) of Rajagiri College of Social Sciences (RIRB 2004).

\section{Consent for Publication}

Not Applicable

\section{Availability of data and materials}

The datasets generated and/or analysed during the current study are available from the corresponding author on reasonable request.

\section{Competing Interests}

The authors declare that they have no competing interests

\section{Funding}

Initial funding for the trial is provided by Rajagiri College of Social Sciences (Autonomous) and UGC-UKIERI (UGC- UKIERI-2016- 17- 089, F. NO. $184-3$ / 2017 (IC)). Funders have no role in the design of the study and collection, analysis, and interpretation of data and in writing the manuscript.

\section{Authors contributions}

SMD conceptualized, designed and drafted the manuscript of the protocol. LS performed the formal analysis. NC and AMB revised the manuscript and contributed to the manuscript and its conclusions. KKS and MKJ have contributed to the discussion and content of the paper. BJ supervised the whole process and contributed significantly towards the paper. All authors read and approved the final manuscript.

\section{Acknowledgements}

The authors wish to thank all the students of the DDUGKY programme of Rajagiri College of Social Sciences who were part of the data collection. Authors also express their gratitude to all the DDUGKY staff who were part of the project in collecting the data and delivering the telephonic intervention. Authors would like to specifically mention Mr Rajeev SR, Coordinator, DDUGKY centre of Rajagiri College for his special effort in coordinating and managing the project within the centre.

\section{References}

1. Zandifar A, Badrfam R. Iranian mental health during the COVID-19 epidemic. Asian J Psychiatr. 2020 Mar 4;51:101990. doi: 10.1016/j.ajp.2020.101990. Epub ahead of print. PMID: 32163908; PMCID: PMC7128485.

2. Campion J, Bhugra D, Bailey S, Marmot M. Inequality and mental disorders: opportunities for action. Lancet. 2013 Jul 20;382(9888):183-4. doi: 10.1016/S0140-6736(13)61411-7. PMID: 23870520.

3. Patel V, Kleinman A. Poverty and common mental disorders in developing countries. Bull World Health Organ. 2003;81(8):609-15. Epub 2003 Oct 14. PMID: 14576893; PMCID: PMC2572527.

4. McManus S, Meltzer H, Brugha T, Bebbington P, Jenkins R. Adult psychiatric morbidity in England. Leeds: The NHS Information Centre for health and social care. 2007. DOI: 10.13140/2.1.1563.5205

5. Lehtinen V, Sohlman B, Kovess-Masfety V. Level of positive mental health in the European Union: results from the Eurobarometer 2002 survey. Clin Pract Epidemiol Ment Health. 2005 Jul 21;1:9. doi: 10.1186/1745-0179-1-9. PMID: 16042763; PMCID: PMC1188064. 
6. WHO. Social Determinants of Mental Health. 2014 [Cited 2020 July 8]. Available from

https://apps.who.int/iris/bitstream/handle/10665/112828/9789241506809_eng.pdf;jsessionid=606C83A6BF373F83055F11BD67255197? sequence $=1$

7. Barik D, Thorat A. Issues of Unequal Access to Public Health in India. Front Public Health. 2015 Oct 27;3:245. doi: 10.3389/fpubh.2015.00245. PMID: 26579507; PMCID: PMC4621381.

8. Harandi TF, Taghinasab MM, Nayeri TD. The correlation of social support with mental health: A meta-analysis. Electron Physician. 2017 Sep 25;9(9):5212-5222. doi: 10.19082/5212. PMID: 29038699; PMCID: PMC5633215.

9. Hoeft TJ, Fortney JC, Patel V, Unützer J. Task-Sharing Approaches to Improve Mental Health Care in Rural and Other Low-Resource Settings: A Systematic Review. J Rural Health. 2018 Dec;34(1):48-62. doi: 10.1111/jrh.12229. Epub 2017 Jan 13. PMID: 28084667; PMCID: PMC5509535.

10. Kroenke K, Spitzer RL, Williams JB. The PHQ-9: validity of a brief depression severity measure. J Gen Intern Med. 2001 Sep;16(9):606-13. doi: 10.1046/j.1525-1497.2001.016009606.x. PMID: 11556941; PMCID: PMC1495268.

11. Zimet GD, Dahlem NW, Zimet SG, Farley GK. The Multidimensional Scale of Perceived Social Support. J Personal Assess. 1988; 52(1): 3041. https://doi.org/10.1207/s15327752jpa5201_2.

12. WHO. Wellbeing Measures in Primary Health Care/The Depcare Project. 1998 [Cited 2020 July 14]. Available from https://www.euro.who.int/_data/assets/pdf_file/0016/130750/E60246.pdf

13. Dambi JM, Corten L, Chiwaridzo M, Jack H, Mlambo T, Jelsma J. A systematic review of the psychometric properties of the cross-cultural translations and adaptations of the Multidimensional Perceived Social Support Scale (MSPSS). Health Qual Life Outcomes. 2018 May 2;16(1):80. doi: 10.1186/s12955-018-0912-0. PMID: 29716589; PMCID: PMC5930820.

14. Topp CW, Østergaard SD, Søndergaard S, Bech P. The WHO-5 Well-Being Index: a systematic review of the literature. Psychother Psychosom. 2015;84(3):167-76. doi: 10.1159/000376585. Epub 2015 Mar 28. PMID: 25831962.

15. Indu PS, Anilkumar TV, Vijayakumar K, Kumar KA, Sarma PS, Remadevi S, Andrade C. Reliability and validity of PHQ-9 when administered by health workers for depression screening among women in primary care. Asian J Psychiatr. 2018 0ct;37:10-14. doi: 10.1016/j.ajp.2018.07.021. Epub 2018 Jul 30. PMID: 30096447.

16. Zimet GD, Powell SS, Farley GK, Werkman S, Berkoff KA. Psychometric characteristics of the Multidimensional Scale of Perceived Social Support. J Pers Assess. 1990 Winter;55(3-4):610-7. doi: 10.1080/00223891.1990.9674095. PMID: 2280326.

17. Laksmita OD, Chung M-H, Liao, Y-M, Chang, P-C. Multidimensional Scale of Perceived Social Support in Indonesian adolescent disaster survivors: A psychometric evaluation. PLoS ONE. 2020;15(3): e0229958. doi:10.1371/journal.pone.0229958

18. Mitchell G, Pistrang N. Befriending for mental health problems: processes of helping. Psychology and Psychotherapy: Theory, Research and Practice. 2011;84(2):151-169. https://doi.org/10.1348/147608310X508566

19. Peardon L, Yellowlees D, Pratt R, et al. The use of innovative methods designed to relieve social isolation in patients with chronic heart failure; volunteer befriending, forums and a newsletter. Eur J Cardiovascular Nurs. 2010 Sep;9(3):181-187. DOI: 10.1016/j.ejcnurse.2009.12.001.

20. Bradshaw T, Haddock G. Is befriending by trained volunteers of value to people suffering from long-term mental illness? J Advan Nurs. 1998 Apr;27(4):713-720. DOI: 10.1046/j.1365-2648.1998.00618.x.

21. Mead N, Lester H, Chew-Graham C, Gask L, Bower P. Effects of befriending on depressive symptoms and distress: systematic review and meta-analysis. Br J Psychiatry. 2010 Feb;196(2):96-101. doi: 10.1192/bjp.bp.109.064089. PMID: 20118451.

22. Siette J, Cassidy M, Priebe SEffectiveness of befriending interventions: a systematic review and meta-analysis. BMJ Open. $2017 ; 7: e 014304$. doi: 10.1136/bmjopen-2016-014304

23. Nelson R. Combating global health worker shortages: task shifting and sharing may provide one solution. Am J Nurs. 2012 Dec;112(12):178. doi: 10.1097/01.NAJ.0000423494.77404.42. PMID: 23190658.

24. Buttorff C, Hock RS, Weiss HA, Naik S, Araya R, Kirkwood BR, Chisholm D, Patel V. Economic evaluation of a task-shifting intervention for common mental disorders in India. Bull World Health Organ. 2012 Nov 1;90(11):813-21. doi: 10.2471/BLT.12.104133. Epub 2012 Sep 14. PMID: 23226893; PMCID: PMC3506405.

25. Mendenhall E, De Silva MJ, Hanlon C, Petersen I, Shidhaye R, Jordans M, Luitel N, Ssebunnya J, Fekadu A, Patel V, Tomlinson M, Lund C. Acceptability and feasibility of using non-specialist health workers to deliver mental health care: stakeholder perceptions from the PRIME district sites in Ethiopia, India, Nepal, South Africa, and Uganda. Soc Sci Med. 2014 Oct;118:33-42. doi: 10.1016/j.socscimed.2014.07.057. Epub 2014 Jul 29. PMID: 25089962; PMCID: PMC4167946.

\section{Figures}




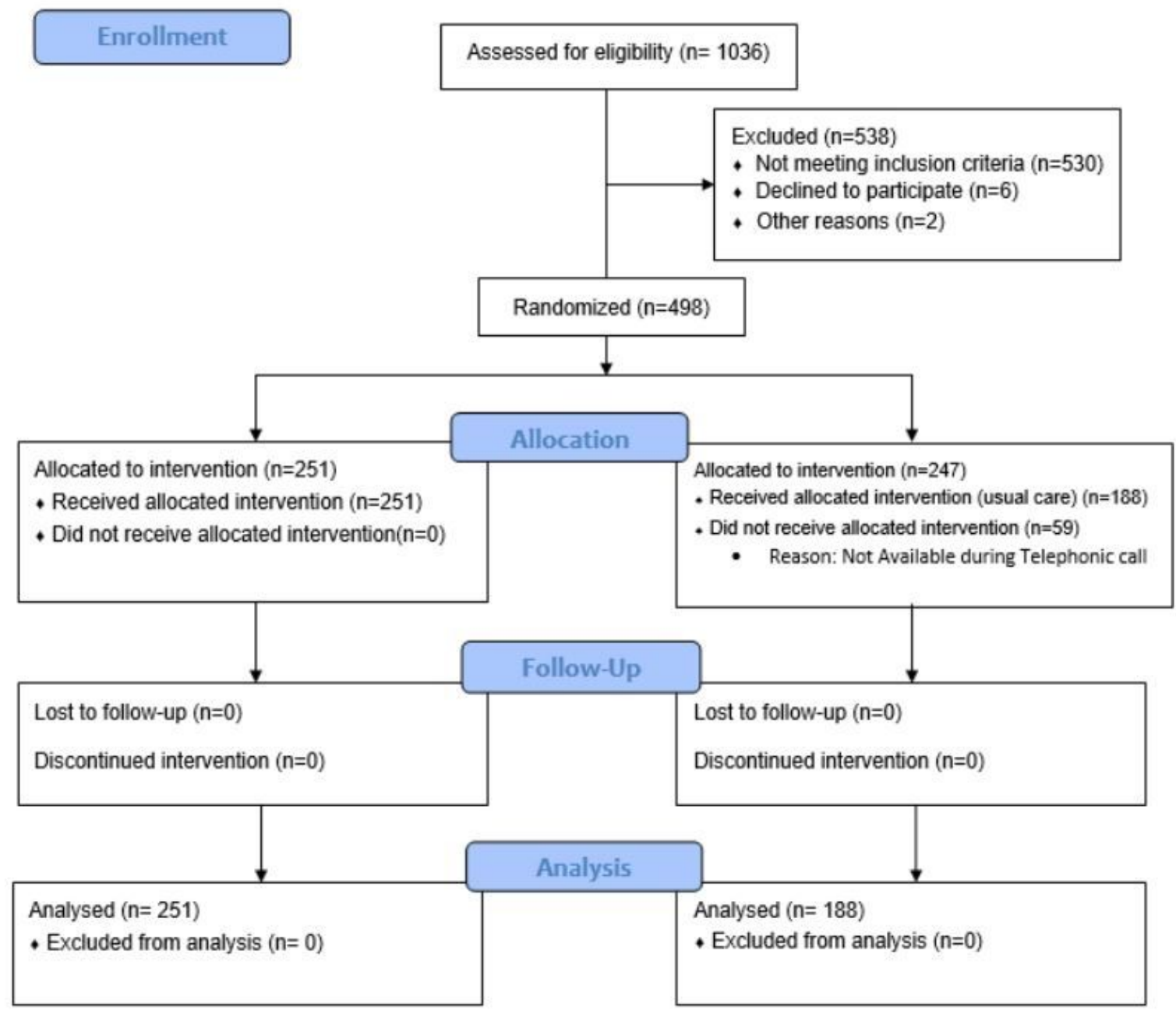

Figure 1

Recruitment of the Participants for the Exploratory Trial. 